\title{
Crecimiento postnatal del recién nacido de muy bajo peso: I crecimiento inmediato
}

\author{
Dra. Mafalda Rizzardini P. ${ }^{\text {; }}$ Dr. Mario Ferreiro S.]; Dra. Patricia Nilo C.1; \\ Srta. María A. Palominos M. ${ }^{2}$; Sr. Samuel Pantoja C. ${ }^{2}$ \\ Postnatal growth of the very low birth weight infant
}

To establish a postnatal growth chart for very low buth weight (VLBW) infants, data obtained from 126 breasi milk fed VLBW patients in actual follow up was studied. For the analysis of resuls infants were divided in three groups accordingly to birth weiglit (BW): A (BW 650 to $1000 \mathrm{~g}$ ); $\mathrm{B}$ (BW 1001 to $1200 \mathrm{~g}$ ) and $\mathrm{C}$ (BW 1201 to $1500 \mathrm{~g}$ ). (jroup A babics lost weight till day 9 of life, group B and C did it only till day 7. Losses amounted to 15.7 , 13.3 and $12.3 \%$ of $\mathrm{BW}$ respectively. BW was again at tained at $25,2 \mathrm{~L}$ and 20 days in the corresponding groups. In all groups $\mathrm{BW}$ was doubled at 70 days of age and tripled at age 100 days. Length increased by 0.66 , 0.65 and $0.63 \mathrm{~cm}$ and hod circunference by $0.65,0.5 \mathrm{I}$ and $0.48 \mathrm{~cm}$ in groups $\mathrm{A}, \mathrm{B}$ and $\mathrm{C}$ in the first eight weeks of life respectively. Although initial increase; in weight, body length and head circunference were similar to figures from other cxperiencies, weiglit incredse after the first two weeks was considered insufficient in these breast milk fed intants. surgesting the need to further evalua te fortified breast milk or hipercaloric artificial formulae for them.

(Kuy words: Very low birth weight, post natal growth, weight, length, head circunference).

El mejor cujdado perinatal e intensivo del prematuro ha atmentado considerablemente el porcentaje de sobrevida de los recién nacidos de muy bajo peso (RVMBP) por lo que adquiere cada vez mis importancia la necesidad de estable. cer patrones de erceimiento postnatal que sirvan para fituras comparaciones y que además, permian una cililicatión más acertada del estado nutrifivo del niño diminu1o a determinada edad.

Durante el último tiempo se ha recomendado algunas curvas de crecimiento post-concepcional de los niños de bajo peso ${ }^{1 \cdot 3}$ pero las causuísticas son ain muy pequeñes, subre todo en los de peso inferior a JoOd g. como para sacar conclusiones delinitivas. Por otro lado. la edad gestacional calculada según fecla de la última menstruación esta sejeta a domasiados errores como parat poder aplicarla en la práctica diaria.

Ll objeto de esta comunicación es dar a onocer algunos indicadores antroponétricos que

1. Departamento de pediatría, Hospital Roherto del Rio, Santiago, Chile.

2. Internos de la Carresa de Medicina. Universidad de Chile. sirvan como guia para la calificación del crecimiento del RNMBP en las primeras semanas de vida extrauterina. En otra oportunidad se darán a conocer los resultados a tres años plazo.

\section{MATIRIAL Y METODOS}

Se estudiaron en forma prospectiva 126 recién naciLos de 680 a $1500 \mathrm{~g}$ que egresaron de la unidad de neonatologia del Hospital Roberto del Rio, entre 1983 y 1987 . Este centro atiende una población urbana de escasos recurses y los nirios nacen en una maternidad estatatal no anexa al Hospital Pediátrico.

Ln el estudio se incluyeron sólo recién nacidos con peso adecuado para la edad gestacional tomando como modelo las curvas de Lubchenco y colaboradores. ${ }^{4} \mathrm{La}$ edad gestacional, salvo en unos pocos casos en que había dingnóstico cuogrático precoz. se calculó por la fecha de li última menstruación. Se descartaron los niños que, por el cxamen físico y comportamiento incial, eran obviamente pequeños para la edad calculada y los con unomalías u olras entermedades congénitas graves. No se excluyeron los prematuros con trastornos propios de sa inmadurez. (síndrome de dificultad respiratoria, persisiencia del ductus arterioso, displasia broncopulmonar) sólo exigiéndose que sobrevivieran hasta el alta deI hospital. La atcrición se hizo en cuna têrnica con servocontrol mientras requirieron cuidado intensivo y luigo en incubatora estándar hasta que fueron capaces de regular la temperatura. El peso se registró en una 
bakanza Seca( anotándose los $10 \mathrm{~g}$ más prúximos. La talla se midió en un pedómetro desde la planta hasta la coronilla y ul petímetro cefálico con una huindia netálica circunstribiendo el cráneo desde el occipucio hasta la región supraciliar.

La alimentacion inicial se indico desplies de 24 y 72 horas, administrándose mientras tanto, por vía intravenosa, solución de gluos sa $5 \%$ en volúmenes iniciales de 70 a $80 \mathrm{ml} / \mathrm{kg} \times$ día, completando el aporte oral de keche, según balan we. Entre el scequnda $y$ cuarlo día (2 a 3 dias en promedio) sc injeió la alimentación con leche humana de banco, mediante sonda nasogástrica intermitente, cada dos horas el comienzo y con pequeños volúmencs, buscando la tolerancia máxima hasta alcanzar afortes de 150 a $180 \mathrm{ml} / \mathrm{kg} \times$ día, lo que se consiguió al linal de la segunda semana de vida. La alimentación oral se interrumpió si aparecían distensiom abdorsimal o tetenciún gástrica. Cuando las condiciones del nuiso lo permitieron sc distanciaron las tomas hasta intervalos de cada tres lroras, por método de gravedad y luego cada cuatro horas, por biberón. [Ina vez que e] peso llegó a $2000 \mathrm{~g}$ se cambió la feche bumana por leche de vaca modificada (NAN (B) con $5 \%$ de dextromultosd.

Para el análisis del material he dividieron los suje tos en tres grupos de acuerdo al peso de nacimiento. Iil grupo A incluyó 30 recién nacidos de $1000 \mathrm{~g}$ o menos. al grupo B, 49 niños de 1001 a 1200 g y el grupo C. 47 pacientes de 1201 a $1500 \mathrm{~g}$. T. la tabla 1 se anotan algunas características de los pacientes.

\section{RESULTADOS}

Los recién nacidos del grupo A perdieron peso hasta el noveno día de vida y los del grupo B y C, hasta el séptimo día. La pérdida máx una porcentual de peso - $\dddot{x}$ (DE)-fue $15,7(7,2) ; 13,2(5,8)$ y $12.3(7,0)$ en los grupos A, B y C respectivamente. El tiempo de recuperación del peso de nacimiento $-x(D E)-$ fue $25,1(9,5) ; 21 \cdot 3(7,2)$ y $20,2(9,6)$ mientras que el necesario para duplicarlos fue $75,7(13,3) ; 72,3(13,7)$ y 71.2 $(15,2)$ y para triplicarlo $106,2(19.4) ; 103,4$ $(15,4)$ y $117,0(18,4)$ dias respectivamente.

Una vez completado el descenso ponderal los recién nacidos del grupo A aumentaron $13,3 \mathrm{~g}$ diarios. durante las primeras 8 semanas, los del grupo B 15,3 y los del grupo C $14,9 \mathrm{~g}$. en total 57,$2 ; 60,1$ y $47,8 \%$ del peso de nacimiento respectivamente.

En la tabla 2 se muestran los pesos de cada grupo en los primeros 56 días de vida y en la figura 1 se comparan con los descritos por Dancis y colaboradores. ${ }^{5}$

El incremento de la talla fue más lento en las dos primeras semanas de vida que en las seis siguientes siendo los promedios para las primeras ocho 0,$66 ; 0,65$ y $0,63 \mathrm{~cm}$ por semana, en $l o s$ grupos A, B y C, respectivamente. El aumento
Tabla 1 .

Características clínjeas de 126 recién nacidos de muy bajo peso, según grupo

\begin{tabular}{|c|c|c|c|}
\hline $\begin{array}{l}\text { Crupo: } \\
\text { Número: } \\
\text { Sexo } \mathbf{H} / \mathbf{M} \text { : }\end{array}$ & $\begin{array}{c}A \\
30 \\
1416\end{array}$ & $\begin{array}{c}B \\
49 \\
20 / 29\end{array}$ & $\begin{array}{c}C \\
47 \\
25 / 22\end{array}$ \\
\hline \multicolumn{4}{|c|}{ Peso nacimiento } \\
\hline E: x (DE) & $950(85)$ & $1121 \pm(53)$ & $1359 \pm(91)$ \\
\hline Rango & $680-1000$ & $1001 \cdot 1200$ & $1201 \cdot 1500$ \\
\hline \multicolumn{4}{|c|}{ I:dad gestacional } \\
\hline Sem: $\dot{x}(D E)$ & $27,1(1,0)$ & $28,5 \pm(1,1)$ & $30,3 \pm(1,2)$ \\
\hline Rango & $27 \quad 29$ & $27 \cdot 31$ & $28 \cdot 33$ \\
\hline \multicolumn{4}{|l|}{ Talla } \\
\hline con: $x$ (DL $)$ & $34,8,5(1,58)$ & $37,37 \pm(1,37)$ & $39,06 \pm(1,66)$ \\
\hline Rango & $31,0 \cdots 37,0$ & $33,5 \quad 40,0$ & $34,0 \quad 42,5$ \\
\hline \multicolumn{4}{|l|}{ P. C'ctálico } \\
\hline $\operatorname{cms}: \bar{x}(D \mid:)$ & $23,78(0,9)$ & $25.77 \leqslant(1,03)$ & $27,72 \pm\{1,6\}$ \\
\hline Rango & $23,0 \quad 26,5$ & $25,0 \quad-28,0$ & $25,0 \cdot 30,5$ \\
\hline
\end{tabular}

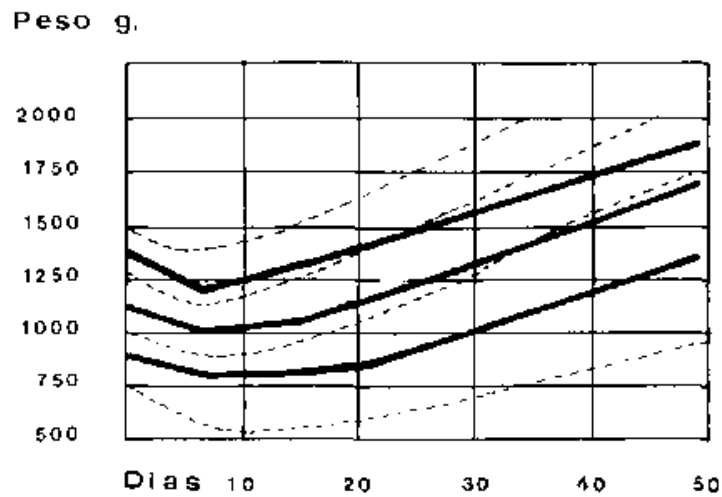

Figura 1. Evolución del peso durante las primeras ocho semanas de vida en recién nacidos de muy bajo peso, lineas enteras. Resultados de Dancis y col. 5 línea cortada.

Tabla 2.

Peso promedio durante las ocho primeras semanas de vida en recién nacidos de muy bajo peso

Peso (g): $\overline{\mathrm{x}}(\mathrm{DF})$

\begin{tabular}{ccccc}
\hline Grupo & A & B & C \\
\hline Semanas & & & & \\
0 & $915(85)$ & $1.121(53)$ & $1.359(91)$ \\
1 & $784(99)$ & $976(96)$ & $1.220(138)$ \\
2 & $826(95)$ & $1.047(101)$ & $1.283(168)$ \\
3 & $902(127)$ & $1.118(105)$ & $1.380(185)$ \\
4 & $970(145)$ & $1.227(130)$ & $1.500(216)$ \\
5 & $1.061(178)$ & $1.360(163)$ & $1.620(228)$ \\
6 & $1.173(2020)$ & $1.495(173)$ & $1.740(223)$ \\
7 & $1.326(238)$ & $1.643(203)$ & $1.844(221)$ \\
8 & $1.438(270)$ & $1.795(218)$ & $2.009(350)$ \\
\hline
\end{tabular}


total de la talla es prácticamente igual en todos los grupos: 5,$27 ; 5,17$ y $5,04 \mathrm{~cm}$ respectivamente, lo que hace que los canales de crecimiento scan paralelos (tabla 3 y figura 2 ).

E1 perimetro cefálico también aumentó más lentamente en las primeras dos semanas de vida pero lucgo sc observó un acelerado crecimiento de la cabeza. especialmente en los niños más pequerios. El aumento semanal del perimetro cefálico fuc de $0.81 ; 0,64$ y $0,60 \mathrm{~cm}$ respectiva. mentc $y$ el crecimiento total, en las 8 semanas: 6,$5 ; 5,1$ y $4,8 \mathrm{~cm}$ (tabla 4). El tamaño de la cabeza de los más pequerios tendió a acercarse al de los otros grupos a partir de la séptina semana de vida (figura 2).

\section{DISCUSION}

Existen muy pocas curvas de crecimiento postnatal inmediato del RNMBP y casi todas están corregidas tomando en cuenta la edad post-concepcional. ${ }^{1 \cdot 3}$ Entre nosotros, y con fines prácticos, parece más útil poder disponer de

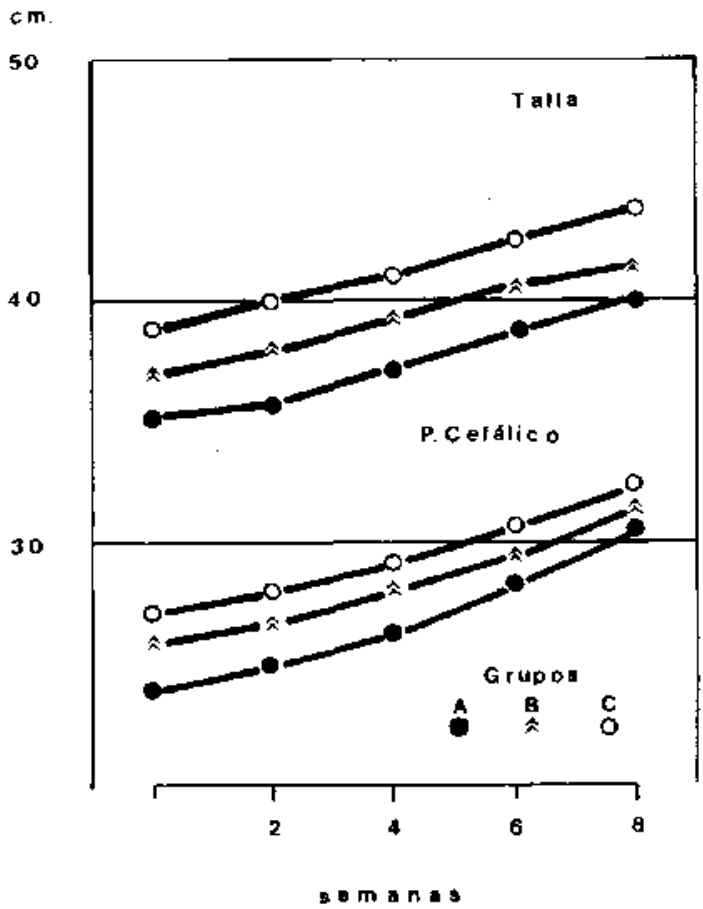

Figura 2. Evolución de la talla y del perímetro cefálico en el recién nacido de muy bajo peso, curante las primeras ocho semanas de vida: Grupo A $(680-1000 \mathrm{~g})$; Grupo B (1001-1200 g); Grupo C (1201-1500 g).
Tabla 3.

Talla promedio durante las ocho primeras semanas te vida en recién nacidos de muy bajo peso

Talla $(\mathrm{cm}): \overline{\mathrm{x}}(\mathrm{DE})$

\begin{tabular}{|c|c|c|c|}
\hline Grupo & A & B & $c$ \\
\hline \multicolumn{4}{|c|}{ Semanas } \\
\hline 0 & $34,85(1,58)$ & $37,37(1,37)$ & $39,06(1,66)$ \\
\hline 2 & $35,65(1,75)$ & $38,10(1,47)$ & $39,95(2,74)$ \\
\hline 4 & $37,10(2,05)$ & $39,47(1,22)$ & $41,42(2,39)$ \\
\hline 6 & $38,82(2,61)$ & $40,75(1,48)$ & $42,77(0,89)$ \\
\hline 8 & $40,12(2,40)$ & $42,54(1,60)$ & $44,10(2,08)$ \\
\hline
\end{tabular}

Tabla 4.

Perimetro cetálico promedio durante las ocho primeras semanas de vida en recién racidos de muy bajo peso

Perímetro cefálico (cm): $\bar{x}$ (DE)

\begin{tabular}{cccc}
\hline Grupo & A & B & C \\
\hline Semanas & & & \\
0 & $23,78(0,90)$ & $25,77(1,01)$ & $27,72(1,60)$ \\
2 & $24,81(1,86)$ & $26,24(0,98)$ & $28,22(2,32)$ \\
4 & $26,48(1,43)$ & $27,64(1,29)$ & $29,67(1,64)$ \\
6 & $28,95(2,02)$ & $29,01(1,96)$ & $30,95(0,79)$ \\
8 & $30,28(1,69)$ & $30,90(1,67)$ & $32,53(2,29)$ \\
\hline
\end{tabular}

algunos estándares de crecimiento postnatal, excluyendo la edad intrauterina, considerando que el cálculo de ésta es poco confiable en un medio con escasa cultura sanitaria y poco acceso al diagnóstico precoz del embarazo.

La meta ideal del crecimiento postnatal es la que simula la del feto en el utero pero ella es difícil de alcanzar porque existe una pérdida inicial de peso que es independiente de la cantidad de liquidos que se administre. ${ }^{6}$

Si se comparan los resultados de esta experiencia con los de Dancis ${ }^{5}$ no se observan grandes diferencias en los primeros dias de vida. A partir de los 20, el aumento de peso de los niños de este estudio es mucho menor. Otros autores ${ }^{7,8}$ encuentran que la velocidad de ascenso ponderal inicial debiera ser mucho mayor que la comunicada por Dancis, especialmente en los RN de menos de $800 \mathrm{~g}$. Si se comparan nuestros hallazgos con los de éstos $y$ otros investigadores ${ }^{7-9}$ no parece haber grandes diferencias por lo menos inicialmente. En todos existe un descenso de peso inicial, mayor mientras más pequeño es el niño, que se prolonga hasta el quinto a noveno día y puede oscilar entre 9 y $21 \%$, con grandes variaciones individuales. Después del nadir, los niños 
aljmentados con dietas hipercalóricas, ${ }^{78}$ con alimentación parenteral total ${ }^{9,10}$ o con leche de su propia madre, ${ }^{11}$ suben más rápidamente de peso y recuperan el de su nacimiento 5 a 10 días antes de lo observado en este estudio. Nuestros resultados podrían sugerir que después de las dos primeras semanas sería conveniente fortificar la leche humana o sustituirla por una fórmula artificial que aporte todos los nutrientes necesarios para un mejor desarrollo.

La talla y el perímetro cefálico del RNMBP, aunque no con la velocidad de la del feto, aumentan desde la primera semana de vida lo que probablemente permita que alcancen los canales normales antes que el peso. El promedio de crecimiento de la talla y del perímetro cefálico resultó mayor que en otras experiencias. Gibbs ${ }^{10}$ observó aumentos de $0,52 \mathrm{~cm}$ por semana en la talla de niños de peso menor a $1000 \mathrm{~g}$ y $0,62 \mathrm{~cm}$ en el perimetro cefálioo, en contraste con 0,66 y $0,81 \mathrm{~cm}$ observados en este estudio.

Estos hallazgos tienen gran importancia porque parece haberse demostrado que e] QI tiene más relación con el incremento inicial del perímetro cefálico y la talla que con el peso ${ }^{12}$ y existe una relación predictiva entre el tamaño de la cabeza y su desarrollo ulterior. ${ }^{13}$

\section{RESUMEN}

Con el fin de establecer una curva de crecimiento postnatal para los RNMBP se estudiaron 126 niños alimentados con leche humanla. Para el análisis de los resultados se dividieron en tres grupos según el peso de racimiento. Grupo A (650 a $1000 \mathrm{~g}$ ). Grupo B (1001 a $1200 \mathrm{~g})$. Grupo C (1201 a $1500 \mathrm{~g})$. Los RN del Grupo A perdieron peso hasta el noveno dia de vida, los de los Grupos B y C, hasta el séptimo dia. El porcentaje de pérdida de peso alcanzó a 15,$7 ; 13,3$ y 12,3 respectivamente. Se recupcró el peso de nacimiento a los $25 ; 21$ y 20 días; se duplicó cerca de los 70 días y se triplicó después de los 100 días. La talla aumentó 0,$66 ; 0,65$ y $0,63 \mathrm{~cm}$ por semana y el perínetro cefálico $0,81,0,64$ y 0,60 por semana en los Grupos A, B y C respectivamente, en las primeras ocho semanas. Los resuita- dos parecen indicar que aunque los aumentos de peso, de talla y del perímetro cefálico inicial pueden compararse con lo observado por otros autores, el incremento ponderal después de las dos primeras semanas sería insuficiente, sugiriendo la necesidad de evaluar el uso de leche humana fortificada o fórmulas hipercalóricas en estos pacientes.

\section{REFERENCLAS}

1. Weldf E., Hering E., Valenzuela B., Anyulo A., Neiro A : Seguimiento de niños con peso de nacimiento inferior a $1500 \mathrm{~g}$. Rev Chil Pediatr 1986; $57: 51-56$.

2. Babson S.: Growth of low birth-weight infants. J Pediatr 1970; 77: 11-18.

3. Gairdner D., Pearson $I$. A growth chart for premature and other infants. Arch Dis Child 1971; 46: $783-787$.

4. Lubchenco L., Housman C.. Boyd E.: Intrauterine growth as estimated from live born birth weight data at 24 to 42 weeks of gestation. Pediatrics $1963: 32: 793-800$.

5. Dancis $J_{\text {., }} O^{\prime}$ Connell $J$.. Hols $L$.: A grid for recording the weight of prematuse infants. 3 Pediatr $1948 ; 33: 570-572$.

6. Fanarof $A$., Hack H.: Fluht requirements of the low-birth weight infant. Rass Conference on Pediatric Research, 1980.

7. Brosius K., Ritter D., Kenny J.: Post natal growth cusve of the infant with entremely low bith weight who was fed enteraly. Pediatrics $1984 ; 74: 778-782$.

8. Shaffer $S$. Quimire Ch., Anderson $J$. Hall $R$.: Postratal weight changes in low birth weight intants. Pediatrics 1987; 79: 702-705.

9. Gill A. Yu $Y$., Bajth B.. Ashuri J.: Postnatal growth in intants born before 30 weeks gestation, Arch Dis Child 1986:61: 549-5.53.

I0. Gibbs $J$ : Routine tolal and supplemental parenteral nutrition for the very low birth weiglt inlant. Ross Conterence on Pediatric Research, 1980.

11. Hodgson M. Rath E., Bruner M., Uauy R. Tomes $f_{\text {.: }}$ Alimentación de recićn nacido de muy bajo peso con leche de su propia madre. Rev Chil Pediatr 1987; 58: 296-301.

12. Rosi $G$. Lipper E., Auld P.. Physical growth and developmental outcorne in very low brith weight premature infants at 3 years age. J Pediatr 1985; 107: 284-286.

13. Gross $S$, Oehler $f$. Eihermon $C:$.: Head growth and developmental outcome in very low birth weight infants. Pediatrics 1983; 71 : 70-75. 